\title{
La autorregulación comunicacional en los procesos de votación: una respuesta jurídico-política al problema del "hacking electoral" practicado a través de las redes sociales. Un análisis del caso colombiano y de su repercusión en América Latina
} Self-Regulation of the Communicational field in the Voting Process: A LegalPolitical Response to the Problem of Electoral Neutrality practiced through Social Networks. An analysis of the Colombian Case and its Impact on Latin America

Submitted: $10 / 12 / 2017$

Revised: 14/01/2018

Accepted: 06/03/2018
Juan Esteban Sanchez Cifuentes*

\begin{abstract}
Purpose - The main proposal of this scientific article is to find some of the shortcomings that may arise from the influence of social networks on the voting decision of Colombian citizens, by reason of the logic of their own democratic system, as well as try to find contributions of the responsive theory to strengthen these fallings.

Methodology/approach/design - To achieve the objective of the research, it is proposed a logical-deductive methodology with a qualitative approach. The main theories on which the research will be based are the systems theory, deliberative theory and responsive theory. The empirical support used is the case studies tool.

Findings - Among the main results obtained with the research are the analysis of the influence of political candidates on the flow of information in social networks. It is recommended that Colombia strengthen its electoral regulatory system.

Practical implications - The main practical contributions of this research are at the political-legal level, since this seeks to strengthen deliberative processes in social networks, and to generate critical awareness and political identity and voting.

Originality/value - This research is original since it analyzes the problem of the influence of the media from another aspect not yet explored sufficiently, from the perspective of the influence of social networks on the decision of citizens to vote, which is why it has a high political and legal value, since it seeks to strengthen the constitutional and democratic order.
\end{abstract}

Keywords: Influence of social networks, Political elections, Responsive regulation, Voting decision, Colombia

\footnotetext{
*Abogado colombiano pela Universidad de Ibagué, Colômbia. Email: esteban.sanchez.cifuentes@gmail.com.
} 


\section{Resumen}

Propósito - El principal propósito de este artículo científico es encontrar algunas de las principales falencias que puedan surgir de la influencia de las redes sociales sobre la decisión de voto de los ciudadanos colombianos, en razón de la lógica de su propio sistema democrático, así como intentar encontrar contribuciones de la teoría responsiva para fortalecer estas falencias.

Metodología - Para lograr el objetivo de la investigación, se propone una metodología lógica-deductiva con un enfoque cualitativo. Las principales teorías sobre las que se fundamentará la investigación serán la de teoría de sistemas, la teoría deliberativa y la teoría responsiva. La herramienta por medio de la cual se le dará soporte empírico será por medio del estudio de casos.

Resultados - Entre los principales resultados obtenidos con la investigación se encuentran el análisis de la influencia de los candidatos políticos en los flujos de información en las redes sociales. Se recomienda que Colombia fortalezca su sistema normativo electoral.

Implicaciones prácticas - Los principales aportes prácticos de esta investigación se encuentran a nivel político-jurídico, ya que esto procura el fortalecimiento de los procesos deliberativos en redes sociales, de formación de conciencia crítica, de identidad política y de votación.

Originalidad / valor - Esta investigación es original puesto que analiza la problemática de la influencia de los medios de comunicación desde un ámbito poco explorado hasta ahora, desde la perspectiva de influencia de las redes sociales sobre la decisión de voto de los ciudadanos, razón por la cual tiene un alto valor principalmente político y jurídico, pues busca el fortalecimiento del orden constitucional y democrático.

Palabras clave: Influencia de las redes sociales, Elecciones políticas, Regulación responsiva, Decisión de voto, Colombia.

\section{Introducción}

Las elecciones políticas en las democracias han tendido a caracterizarse históricamente por presentar un componente competitivo, puesto que, por regla general, es por virtud de la dinámica electoral que en las democracias se define la persona que va a detentar el poder político, sin embargo, en el siglo XX tras la diseminación de la Internet como medio de comunicación comercial se han producido diferentes transformaciones en el terreno político (DUTTON et al., 2010).

Uno de los más plausibles cambios está relacionado con los modos de comunicación política y el debate público que ahora se hacen presentes en las redes sociales y a los cuales se han adaptado viejas estrategias políticas de difamación y de manipulación, además de esto, debido al potencial de 
conectividad que tienen las redes sociales, éstas son prácticas que se han comenzado producir a una escala cada vez mayor (FGV DAPP, 2017).

Con los nuevos espacios que se producen con los medios de comunicación nacen nuevos actores políticos fundamentales en las democracias como lo fueron en su momento, el aparecimiento de empresas públicas y privadas de prensa, radio y televisión. Ahora, tras el surgimiento comercial de la Internet, han comenzado a aparecer nuevos actores en el escenario político como los hackers, quienes están fungiendo un rol fundamental en la política, pero entonces ¿quiénes son los hackers y cuál es su influencia sobre la política?

De cierta forma la palabra hacker es un término problemático, pues no existe una institución formal que haya asumido la responsabilidad de delimitar el alcance de este concepto, tampoco es un término que haya sido acuñado tradicionalmente. La palabra hacker puede ser entendida como un término que responde tanto al movimiento cultural que surgió del contexto histórico que produjo Internet, como también puede ser utilizado como el apelativo que se otorga a aquellas personas que tienen los conocimientos necesarios para manipular sistemas informáticos y que probablemente se ha formado en medio del ambiente colaborativo que ha producido la misma Internet (WIKIPEDIA, [s.d.]).

Asimismo, es necesario indicar que existen diferentes tipos de hackers dependiendo del objetivo con que utilicen sus conocimientos. Así pues, aquellos que utilizan sus conocimientos de forma positiva, generalmente para hallar vulnerabilidades de algún sistema informático en procura de fortalecerlo se denominan "whitehackers", "whitehats" o "hackers éticos", por el contrario, aquellos que utilizan sus conocimientos para dañar a los demás o para obtener beneficios en perjuicio de otros, reciben la denominación de "blackhackers", "blackhats" o "crackers" (INSTITUTO NACIONAL DE TECNOLOGIAS DE LA COMUNICACIÓN, 2012).

De la encrudecida rivalidad entre candidatos políticos en las democracias en la era digital, de los compromisos políticos que son realizados en campaña, de los intereses económicos en juego y de la procura de los candidatos por obtener una ventaja competitiva sobre sus contrarios nace la necesidad de optimizar el uso que los candidatos políticos dan a las redes sociales al ser uno de los principales canales de información de los ciudadanos, con esa empresa, es que los hackers son incluidos en la dinámica de las campañas políticas, como los individuos con los perfiles idóneos para adelantar dicha labor (esto no quiere decir, que sea solamente un hacker quien puede desempeñar esta función en una campaña electoral, pues otro perfil profesional podría cumplirla también) (BONAÑO, 2015; PANTOJA, 2011). 
El arte de la manipulación de los grupos sociales a través de los medios de comunicación digital es real y es una práctica que lleva desarrollándose varios años bajo diferentes movimientos científicos, entre ellos, uno de los más destacados es el de la ingeniería social. En el área de la ingeniería social, uno de los principales exponentes a nivel internacional es Kevin Mitnick, un hacker que llegó a ser uno de los de mayor reconocimiento en el mundo y se especializó en violar la seguridad de sistemas informáticos por medio de la mezcla de técnicas tradicionales de hacking con estrategias de engaño y persuasión (MITNICK; SIMON, 2007). Una de sus obras más importantes “The art of deception" o en su versión en español "El arte del engaño".

Con Internet cada ordenador puede o no ser potencialmente riesgoso para la democracia, pues esto depende tanto de aspectos políticos como jurídicos, ya que la regulación de la Internet está condicionada por la posición regulatoria que asuma el Estado, en razón a que la reglamentación de esta es un asunto complejo, debido, entre otros factores, como lo son la libertad de expresión y el principio de neutralidad de la red, dichos preceptos son esenciales, puesto que mantienen inerme la naturaleza de la Internet y limitan los abusos tanto públicos como privados.

Entre los temas problemáticos que han emergido de las transformaciones producidas por las nuevas tecnologías de la información y de la comunicación, se pueden enunciar entre otros el de la inteligencia artificial, el de la big data y el de las fake news (FRAZÃO, 2017). Por este motivo, lo que se propone hacer a través de esta investigación, es abordar estos temas analizándolos desde la perspectiva de aquellos actores que se encuentran tras la utilización de este tipo de innovaciones tecnológicas para influir en la decisión de voto de los ciudadanos de forma irregular, así como en algunas de las repercusiones políticas y jurídicas de sus actos.

De este modo, el objetivo principal de este artículo es contribuir con el fortalecimiento de la democracia en Colombia, especialmente frente a la regulación de la influencia ilegitima de actores sobre la decisión de voto de los ciudadanos a través de las redes sociales.

Con el propósito de alcanzar este objetivo, la principal pregunta de investigación que se procura resolver con este estudio es la siguiente: ¿qué mudanzas están produciendo las redes sociales en la dinámica electoral colombiana que afectan su orden democrático y cómo este puede verse fortalecida por la teoría responsiva?

Para dar respuesta al interrogante planteado, la investigación se desarrollará en tres partes, una primera parte dirigida a realizar el análisis de un caso que refleja en gran medida la situación política y jurídica que enfrentan las democracias latinoamericanas, frente a los cambios que están produciendo los 
medios de comunicación digital, posteriormente, se realizará un acercamiento a la normativa electoral colombiana para evaluar posibles falencias, finalmente, se analizará como el modelo responsivo puede contribuir al fortalecimiento de la democracia en Colombia.

La hipótesis que se pretende probar con este estudio, es que Colombia no se ha preparado de forma adecuada para asumir los diferentes desafíos democráticos que presentan los nuevos espacios de comunicación digital, razón por la cual, las instituciones se han visto afectadas y los derechos y garantías de los ciudadanos de carácter democrático se han deteriorado.

La metodología empleada para el desarrollo de esta investigación será de tipo lógico deductivo, con un abordaje cualitativo, pues por medio de ésta se pretende identificar y comprender los signos y símbolos de la realidad política colombiana, para de esta forma, por medio de la observación y posterior conocimiento del problema, se puedan inferir algunas recomendaciones.

\section{El caso colombiano: Blackhackers sobre las elecciones políticas, una sombra que se extendió por América Latina}

Los comicios electorales en las democracias occidentales se han caracterizado por la competitividad y por la libertad con que se enfrentan sus candidatos políticos. La libertad desde sus diferentes aristas es un derecho reconocido por gran parte de los Estados de corte occidental, así como también, es considerado por diferentes doctrinarios como uno de los valores más esenciales en la configuración de los Estados Sociales de Derecho (CARBONELL, 2008).

Con la influencia del constitucionalismo y neoconstitucinonalismo como importantes movimientos político-jurídicos que definieron en gran medida los flujos de la política y el derecho, después de las crisis producidas con la I y la II Guerra Mundial, fueron ampliamente limitados los poderes del Estado y se otorgaron mayores garantías a los ciudadanos, fortaleciendo de esta forma la libertad como uno de los valores esenciales en la configuración del orden social en occidente (COMANDUCCI, 2002). En este aspecto, durante años ha sido notable la influencia del modelo económico, político y jurídico norteamericano (GANDÁSEGUI et al., 2016; NIETO, 2010).

En este sentido, Colombia como uno de esos países cimentados sobre un sistema de orientación constitucional y democrática no se escapa de las competencias electorales por el poder político. En este aspecto, los medios de comunicación colombianos son fundamentales, pues según lo señala Manuel Castells (2009) los medios de comunicación son los principales intermediarios en los procesos de construcción de significado social, ya que se ocupan de una labor 
esencial en las campañas políticas, en razón a que asumen la labor de transmitir la información electoral a los votantes, motivo por el cual modelan la visión que los ciudadanos tienen de su entorno.

Al analizar el recorrido político que viene llevando Colombia, se hace evidente el complejo momento histórico por el que atraviesa dentro del contexto latinoamericano, especialmente si se evidencia que Colombia enfrenta un ambiente hostil desde hace más de 50 años debido a la terrible lucha que adelanta contra el narcotráfico y que ha dejado un saldo de 1.754 víctimas de violencia sexual, 10.456.552 millones de refugiados (al año 2012) y 220.000 víctimas mortales (al año 20143) (CENTRO NACIONAL DE MEMORIA HISTÓRICA, 2012; EL PAÍS, 2013).

Para conseguir dimensionar la complejidad que involucra el conflicto armado en Colombia se puede señalar que tan solo para el año 2002, es decir, hace más de 15 años, el patrimonio bruto anual de las FARC $^{1}$ aumentaba aproximadamente 2.000 millones de dólares por año, según cifras del Ministerio de Defensa de Colombia (EL TIEMPO, 2002).

Después de examinar algunas de las cifras que reflejan lo que ha sido la guerra en Colombia, es importante denotar que a pesar de que son diferentes las voces que indican que los motivos de la guerra son políticos, tras evidenciar estos datos, se puede inferir que además de intereses políticos se encuentran ocultos fuertes intereses económicos, más aún, si se tiene en cuenta que el país atraviesa por un periodo de transición tan importante como lo es el del posconflicto, luego de la firma de un tratado de paz con la guerrilla de las FARC, motivo por el cual, diferentes cuestiones sociales, políticas y económicas se encuentran por definir.

Con relación al tema del conflicto armado y el Proceso de Paz en Colombia uno de los casos más famosos y controversiales fue el de Andrés Fernando Sepúlveda, un hacker $^{2}$ de origen colombiano que fue investigado y condenado por adelantar operaciones de manipulación de elecciones políticas en diferentes países de América Latina a través de redes sociales principalmente.

Este hombre reconoció personalmente a la revista Bloomberg Businessweek haberse dedicado durante un 8 años a interferir en los comicios electorales de países como Nicaragua, Panamá, Honduras, El Salvador, Colombia, México, Costa Rica, Guatemala y Venezuela en favor de los intereses políticos particulares que lo financiaban (BLOOMBERG BUSINESSWEEK, 2016).

\footnotetext{
${ }^{1}$ El significado de la sigla corresponde a las Fuerzas Armadas revolucionarias de Colombia, una de las guerrillas más fuertes y de mayor tradición en el Continente Americano.

${ }^{2}$ El termino hacker debe ser entendido como la persona que cuenta con los conocimientos técnicos para hallar y aprovechar las fallas de un sistema informático. Asimismo, el termino black-hacker debe ser entendido como aquella persona que utiliza sus habilidades técnicas para beneficiarse en perjuicio de otros. 
Específicamente en Colombia, el hacker fue vinculado con el desarrollo y ejecución de propaganda negra, la cual, incluía hurto de datos personales e interceptaciones ilegales, entre otras prácticas relacionadas con sus habilidades de hacking. Sus actividades en Colombia, según lo señaló el propio Sepúlveda, fueron promovidas por influyentes políticos colombianos, que entre otras instrucciones le ordenaban oponerse al gobierno del entonces presidente Juan Manuel Santos, atacar a través de medios digitales a aquellos que apoyaban el Proceso de Paz y obtener información privilegiada de los representantes de las FARC en las mesas de negociaciones del Proceso de Paz en la Habana-Cuba.

Por estos hechos, el hacker Sepúlveda fue condenado en Colombia en el año 2015 a pagar 120 meses de prisión y 120 salarios mínimos mensuales legales vigentes de Colombia, por los delitos de concierto para delinquir, acceso abusivo a sistema informático, violación de datos agravado, uso de datos maliciosos de instrumento privado y espionaje, en contra de los miembros negociadores del Proceso de Paz que representaban a las FARC en la Habana - Cuba y del exvicepresidente Francisco Santos (FISCALIA GENERAL DE LA NACIÓN, [s.d.]).

Con relación al singular comportamiento que se presentó en las redes sociales en momentos de agitación política en Colombia como los que se observaron con el Plebiscito por la Paz, fueron múltiples las denuncias que realizaron diferentes medios de comunicación, con relación a posibles interferencias ilegitimas para manipular la información que en ese momento circuló por la red (EL TIEMPO, 2016; LA REPÚBLICA, 2016; RAZÓN PÚBLICA, 2016; SEMANA, 2016).

Entre las principales estrategias que fueron denunciadas por los medios de comunicación para ser utilizados con el fin de influir en los votantes se encuentran los rumores y las fake news, entre los que se destacaron, la supuesta "imposición de aquello que se denominó como la ideología de género que sería impartida en los colegios", "la presunta alteración de resultados electorales por medio de la utilización de tinta que borrable en los tarjetones" y la incertidumbre por la supuesta "implementación del modelo de socialismo venezolano en Colombia", entre otras informaciones que resultaron ser falsas (EL TIEMPO, 2016; RAZÓN PÚBLICA, 2016; SEMANA, 2016).

Ahora bien, con el objetivo de facilitar el análisis de las diferentes actividades practicadas por el hacker puede realizarse una distinción relacionada con el tipo de herramientas que utilizó y con relación al objetivo que perseguía con cada tipo de operación, pues cada una de estas modalidades produjo efectos diferentes y amerita una perspectiva de estudio diferente.

Por un lado, se encuentran aquellas prácticas que buscaban atentar contra la seguridad informática de diferentes personas e instituciones con el objetivo de 
recabar información privilegiada y privada. Estas operaciones dependían en gran medida de la utilización de técnicas típicas de hacking ${ }^{3}$ como lo son el spoofing ${ }^{4}$, el sniffing ${ }^{5}$, los MitM ${ }^{6}$, los malware y $^{7}$ los Dos o DDos ${ }^{8}$.

Por otro lado, se encuentran las actividades que buscaban alterar la opinión pública, pues una vez que el equipo de Sepúlveda realizaba el proceso de recolección de información de diferentes actores sociales y políticos, el paso a seguir era la manipulación de los datos y su publicación en las redes sociales, valiéndose de herramientas como los bots, perfiles falsos o robados, rumores y fake news, entre otras técnicas de manipulación, con el objetivo de generar la atmosfera propicia para influenciar a los ciudadanos.

Estas dos clases de procedimientos ameritan una especial atención y deben ser igualmente atendidas por las autoridades competentes, con el objetivo de resguardar y garantizar los derechos y las prerrogativas democráticas de los ciudadanos, sin embargo, las prácticas de alteración a la opinión pública son probablemente las de mayor impacto social, político y económico, puesto que es por medio del sufragio que se garantiza la distribución del poder político, se legitima la actuación del gobierno y se eligen representantes para que asuman las decisiones administrativas en las democracias.

\footnotetext{
${ }^{3}$ No obstante la aparente novedad de este tipo de procedimientos en las campañas políticas, es importante evidenciar que estos procedimientos no son del todo innovadores, puesto que estas prácticas no se alejan demasiado de antiguas estrategias políticas como aquellas que fueron develadas con el controversial caso del Watergate, puesto que se basaban en la violación de un medio de comunicación que se suponía privado. Este fenómeno en la era digital ha sido estudiado desde diferentes ámbitos, como, por ejemplo, a través del derecho penal por medio de teorías como la de los delitos informáticos. Fue a través de la ley 1273 de 2009 que se modificó el Código Penal de Colombia para incluir un nuevo conjunto de tipos penales dirigidos a sancionar los delitos informáticos.

${ }^{4}$ El spoofing envuelve algún tipo de falsa representación de información hecha por la victima (BABU, R; BHASKARI, L; SATYANARAYANA; CH. A Comprehensive Analysis ofSpoofing. International Journal of Advanced Computer Science and Applications, [S.1.] vol. 1, n.6 p. 152, dez. 2010).

${ }^{5}$ El sniffing es una técnica que permite captura y monitorear el tráfico que circula por una red (SPANGLER, R. Packet Sniffer Detection with AntiSniff. Wisconsin: University of Wisconsin, 2003).

${ }^{6}$ Los MitM o hombre en el medio, tiene el objetivo de ganar el acceso a la información de la víctima a través de la interceptación de la comunicación (BOND, M.; ANDERSON, R. The Man-in-the-Middle Defence. 2009).

${ }^{7}$ Los malware son componentes de software puestos intencionalmente en un sistema para provocar daños o violar su seguridad (BILEY, M. et al. Automated Classification and Analysis of Internet Malware. Michigan: University of Michigan, 2007).

${ }^{8}$ Los ataques de denegación de servicio tienen como objetivo la interrupción de la transmisión de la información a través de una cantidad excesiva de solitudes de servicio (MIRKOVIC, J. REIHER, P. A Taxonomy of DDoS Attack and DDoS Defense Mechanisms. Computer Communications Review, Los Angeles, vol. 34, p. 39-54, abr. 2004).
} 
De este modo, si se profundiza en la lógica de los Estados Democráticos con relación a la manipulación de la opinión pública a través de las redes sociales, quien se vale de maniobras fraudulentas para alterar la voluntad de voto de los ciudadanos desconoce el interés general en beneficio de sus propios intereses particulares atentando contra el orden público, lo que consecuentemente lleva al desconocimiento de los elementos esenciales sobre los cuales descansan el constitucionalismo y la democracia. Sobre este aspecto el artículo $1^{\circ}$ de la Constitución Política de Colombia advierte sobre la configuración del Estado Colombiano que:

"Colombia es un Estado social de derecho, organizado en forma de República unitaria, descentralizada, con autonomía de sus entidades territoriales, democrática, participativa y pluralista, fundada en el respeto de la dignidad humana, en el trabajo y la solidaridad de las personas que la integran y en la prevalencia del interés general" (COLOMBIA, 1991).

Este conjunto de conceptos y enunciados normativos permiten deducir que los actos que están siendo estudiados en este caso están contribuyendo a la desarticulación de elementos primordiales para la Democracia Colombiana como lo son el interés general, la legitimidad, la participación ciudadana, la pluralidad y la representatividad. Dicha desarticulación puede llevar a señalar que, desde la perspectiva política y según los preceptos normativos que configuran la organización estatal de este país latinoamericano, una interferencia tan temeraria sobre los ciudadanos constituye una afrenta contra el orden político y jurídico de Colombia.

Frente a este caso parece haberse producido una fuerte respuesta social e institucional con la conmoción pública y con la condena impuesta al hacker, no obstante, existen aún importantes componentes del caso que parecen pasar desapercibidos bajo la condena de Sepúlveda.

De analizarse el caso con mayor detenimiento, se puede apreciar que hasta el momento, en América Latina son pocos por no decir que nulos los eventos en donde fueron responsabilizados formalmente otros partícipes de los delitos electorales relacionados, por ejemplo, con la dirección y financiación de las operaciones.

De alguna manera, parece como si la condena de los hackers como Sepúlveda hubiese funcionado como un placebo para aliviar la controversia que generó el escándalo por las operaciones, disimulando ante los ojos de la opinión pública latinoamericana que existió una organización a nivel internacional dedicada a la manipulación de los flujos de información política en las redes sociales. 
En otras palabras, existe algo más que evidenciar con el estudio del caso y esto es que la condena que fue impuesta al hacker se realizó por delitos que atentaban contra la seguridad de la información y de los datos y por delitos que atentaban contra la seguridad del Estado, no obstante, a pesar de la notable responsabilidad política que se derivaba de sus actos y del daño causado al sistema electoral colombiano (según fue abordado con antecedencia en este estudio), Sepúlveda no fue condenado por ningún delito que atentara contra los mecanismos de participación democrática.

Esto significaría que, de ser investigado el hacker por estas conductas punibles, deben ser vinculados a la investigación todos aquellos que hubiesen participado de los delitos que afectaron los mecanismos de participación democrática, incluyendo a aquellos candidatos políticos que se hubiesen beneficiado directa o indirectamente con los resultados de sus operaciones.

No es secreto el hecho de que algunos de los principales interesados en los resultados de las elecciones políticas son los mismos candidatos, pues estos son los directamente beneficiados, así como tampoco, es un secreto que en virtud de esos intereses prácticas como las del Watergate se han extendido por las democracias para asegurar que algunos candidatos obtengan una ventaja competitiva sobre sus contendores políticos (CASTELLS, 2009; ROBINSON, 1974).

En este aspecto, ni los presuntos autores de los delitos que atenten contra los mecanismos de participación democrática en Colombia, que para este caso, podrían ser aquellos que llevaron a cabo las operaciones, ni tampoco las personas que coordinaron, dirigieron y financiaron sus actividades están asumiendo alguna responsabilidad con el sistema electoral, lo cual, llevaría a inferir que existe una omisión por parte del Estado frente a la obligación que tiene de salvaguardar del orden electoral e impartir justicia, no obstante, asumir esta determinación corresponde exclusivamente a las autoridades competentes.

Es de señalar que, en Colombia por lo menos al finalizar el año 2017 no se ha presentado algún caso en donde una figura política haya asumido algún tipo de responsabilidad o haya sido condenada por los hechos vinculados con el caso Sepúlveda.

Una vez agotada la primera parte del estudio, con relación a la implicación de los hackers en episodios recientes de la política colombiana y analizadas algunas de sus repercusiones en aspectos democráticos, resta por explorar un asunto fundamental y este es el de la responsabilidad que tienen los aquellos que dirigieron y financiaron las actividades de los hackers. 


\section{Colombia bajo la óptica electoral: la responsabilidad por la influencia ilegitima en la decisión de voto de los ciudadanos a través de las redes sociales}

Con relación a la normativa que regula la organización electoral en Colombia, se puede señalar que una de las normas fundamentales es el Código Electoral, Decreto 2441 de 1986. Este Decreto ha sido objeto de continuos cambios a través de reformas constitucionales y leyes de la república, que si bien, han contribuido con el desarrollo y mejoramiento del mismo, son modificaciones que no se han articulado de forma adecuada dentro de un esquema coherente (LEGISLATIVO, 2009).

El Código Electoral Colombiano, el cual, debería ser la piedra angular del sistema electoral, se encuentra parcialmente vigente en la actualidad debido principalmente a que no fue adaptado para incorporar los diferentes cambios que en materia electoral fueron introducidos al ordenamiento jurídico por la Constitución Política de 1991 (LEGISLATIVO, 2009).

Asimismo, con relación a las autoridades que se encargan de proteger el orden electoral colombiano y de vigilar a los actores que compiten en las elecciones políticas, se encuentran dos principales autoridades de rango constitucional, estas son, el Consejo Nacional Electoral (CNE) y la Registraduría Nacional del Estado Civil (RNEC). ${ }^{9}$ Estas autoridades son órganos públicos que tienen la obligación de garantizar los derechos y garantías democráticas de los ciudadanos.

De conformidad con lo anterior, Colombia como un Estado social y democrático de derecho, tiene la obligación de garantizar el bienestar social, de velar por la conservación de las garantías democráticas y de procurar la materialización de los derechos humanos y de los derechos fundamentales, particularmente, en asuntos de orden público como aquellos vinculados con el sistema electoral.

En este sentido, según la doctrina de derecho internacional público sobre los derechos humanos, el derecho de libertad de expresión tiene dos aristas diferentes, la primera, se presenta bajo la perspectiva desde quien informa, que es quien tiene el derecho de expresarse de forma autónoma y sin censura, sin perjuicio de la responsabilidad ulterior que se produzca por las declaraciones que emita en virtud de su responsabilidad social como comunicador $y$, la segunda, desde la perspectiva de quien es informado, que es quien tiene el derecho a recibir información de forma veraz e imparcial (BOTERO et al., 2011; CARBONELL, 2008; DUTTON et al., 2010).

\footnotetext{
${ }^{9}$ Instituciones que son consagradas por en el artículo 120 de la Constitución Política de Colombia. 
La Constitución Política de Colombia consagra en su artículo 20 el derecho a la libertad de expresión en los siguientes términos:

"Se garantiza a toda persona la libertad de expresar y difundir su pensamiento y opiniones, la de informar y recibir información veraz e imparcial, y la de fundar medios masivos de comunicación.

Estos son libres y tienen responsabilidad social. Se garantiza el derecho a la rectificación en condiciones de equidad. No habrá censura." (COLOMBIA, 1991)

El anterior postulado normativo establece que el receptor de la información tiene el derecho de ser informado de forma veraz e imparcial, más aún, si se tiene en cuenta, que se trata de asumir una decisión política primordial para la democracia como lo es la de ejercer el derecho al voto, condición que puede estar siendo vulnerada por actividades como las de hacking en campañas de elecciones políticas.

De este modo, las principales herramientas coercitivas con que cuentan los órganos electorales en Colombia, como el Consejo Nacional Electoral y la Registraduría Nacional del Estado Civil, para encausar el comportamiento de los regulados son de naturaleza administrativa, sin perjuicio de lo anterior, con el fin de garantizar la integridad del sistema electoral colombiano y, con ello, salvaguardar los derechos humanos de los ciudadanos, la normativa electoral se apoya en la legislación penal para la protección de los mecanismos de participación democrática a través de los denominados delitos electorales.

Con relación al escenario factico que se viene analizando en este estudio sobre el denominado caso Sepúlveda, se puede advertir que existe un tipo penal en el ordenamiento jurídico colombiano que protege los mecanismos de participación democrática y que podría describir la conducta de aquellos que participaron en las operaciones de hacking; esta norma correspondería al delito del fraude electoral, el cual, se encuentra consagrado en el artículo 388 del Código Penal Colombiano, que indica:

"El que mediante maniobra engañosa obtenga que un ciudadano o un extranjero habilitado por la ley, vote por determinado candidato, partido o corriente política o lo haga en blanco, incurrirá en prisión de cuatro (4) a ocho (8) años, y multa de cincuenta (50) a doscientos (200) salarios mínimos legales mensuales vigentes.

En igual pena incurrirá quien por el mismo medio obtenga en plebiscito, referendo, consulta popular o revocatoria del mandato votación en determinado sentido. 
La pena se aumentará de una tercera parte a la mitad cuando la conducta sea realizada por un servidor público". (Colombia, 2000)

Entre otras apreciaciones del tipo penal antes citado, se puede mencionar que posee un verbo rector determinado que es "obtener" 10 , el bien jurídico tutelado por el Estado a través de este tipo penal es la "participación democrática", el sujeto activo es indeterminado, el sujeto pasivo es de forma principal la persona que es titular del derecho de sufragio y de forma secundaria el Estado como garante de la organización electoral y, finalmente, se encuentran contempladas la multa y la prisión como consecuencias jurídicas para quien transgrede la norma.

Bajo estas condiciones normativas y con base en el previo análisis del caso Sepúlveda, es de señalar, que pudo llegarse a producir una vulneración de los mecanismos de participación democrática y, que según la teoría de la participación en los delitos, los hackers podrían ser presuntamente responsables en calidad de autores por el delito de fraude al elector, mientras que las personas que hubiesen dirigido, financiado y se hubiesen beneficiado con el resultado de sus operaciones, serían presuntamente responsables en calidad de determinadores. ${ }^{11}$

De lo anterior, se colige que aquellas personas que participaron presuntamente en la comisión de delitos electorales a través de las operaciones de hacking, son responsables penalmente, sin perjuicio de la responsabilidad administrativa o disciplinaria que se llegase a imponer (esta última, recae sobre las personas que ostenten la calidad de servidores públicos).

Empero, a pesar de las prerrogativas normativas existentes, de la gran estructura del aparato burocrático colombiano en aspectos electorales y de la aparente acción del Estado, lo evidenciado con este estudio, es que los hackers actuaron en contra del orden electoral con gran libertad y aun nadie ha asumido

\footnotetext{
${ }^{10}$ Cabe agregar que para la adecuación de la conducta típica es necesario que se dé el depósito efectivo de la tarjeta electoral en la urna para que de manera efectiva el ciudadano adquiera la calidad de sufragante.

${ }^{11}$ Según la explicación de la teoría de la participación de Alfonso Reyes Echandía "el determinador es la persona que induce a otro a que realice un hecho punible, siendo esta una forma de coparticipación que necesita la presencia de dos tipos de sujetos: el determinador, quien proporciona la idea criminal y la transmite, o fortalece la idea que apenas nacía en mente ajena. El segundo sujeto es el ejecutor material, quien convierte en comportamiento típico la idea, de manera que este es el único y verdadero autor, mientras que el determinador es el ideador u orientador del hecho punible" (Huertas, Amaya y Malte, 2013, Opinión Jurídica, Vol. 12, N²3, pp. 83 - ISSN 1692-2530 • En 81 ero-Junio de 2013 / p.212 Medellín, Colombia).
} 
la responsabilidad sobre la afectación a los mecanismos de participación democrática, bien jurídico que debe ser protegido por el Estado.

La ausencia de responsabilidad anteriormente señalada conlleva a la inferencia de que las entidades que en Colombia tienen la función de velar por la salvaguarda de los derechos electorales no la están haciendo satisfactoriamente, pues probablemente no cuentan con los instrumentos jurídicos necesarios o de contar con dichos instrumentos, estos no son lo suficientemente eficaces para hacerlo.

Una vez se lograron identificar algunas de las falencias en el sistema electoral colombiano y se definieron algunas de sus características, se pasará a analizar como la teoría responsiva puede contribuir con su fortalecimiento.

\section{Teoría responsiva, un complemento a la tradicional forma de regulación electoral}

La teoría responsiva es una corriente regulatoria novedosa que propone replantear elementos del modelo de regulación tradicional. Esta teoría parece desde diferentes ámbitos atractiva, puesto que, entre otros aspectos complementa falencias existentes en los modelos tradicionales de regulación, debido a que ofrece una formidable fundamentación que integra el derecho, la economía y la política, no obstante, antes de pasar a analizar el fundamento de esta teoría, se realizará un acercamiento a algunos de sus principales presupuestos, para posteriormente, proponer recomendaciones que puedan contribuir al fortalecimiento de la democracia, con base en las falencias que fueron halladas en el sistema democrático colombiano a partir del estudio del caso Sepúlveda.

De esta forma, uno de los principales presupuestos de la teoría responsiva tiene su origen en la teoría de sistemas, específicamente en el concepto de autopoiesis. El concepto de autopoiesis fue trasladado a la teoría jurídica por Niklas Luhmann. Este autor se apoyó en los avances científicos de los biólogos Maturana y Varela para explicar según él, como el derecho es independiente y se autocompone al igual que lo hacen los sistemas biológicos (ANTUNES, 1989). Para Luhmann (2003), la autonomía es una condición de existencia de los sistemas sociales al igual que los biológicos.

Lo que básicamente buscaba argumentar el sociólogo con el concepto de autopoiesis, es que todos los sistemas son independientes pues cuentan con los recursos para autocomponerse y subsistir por su cuenta, motivo por el cual, es dificultoso y desgastante intentar una interferencia directa de un sistema sobre otro, en razón a que cada sistema es independiente y la influencia directa de un sistema sobre otro reduciría sus posibilidades de sinergia y produciría mayor resistencia (ANTUNES, 1989; LUHMANN, 2003). 
En palabras de Maturana y Varela citadas por Antunes "la autopoiesis en el espacio físico constituye la condición ultima, necesaria y suficiente de la propia vida" (1989). Esto significa que todo sistema, sea este de naturaleza social o biológica cuenta con los elementos necesarios para subsistir y ser autónomo.

Por otra parte, si se analiza el papel del Estado regulador a la luz del concepto de autopoiesis, se puede colegir que tanto el Estado como los regulados, con dificultad responderán a estímulos que se les inflijan en forma directa, esto quiere decir, que intentos bruscos y directos de regulación de un sistema sobre otro, como los que se realizan con los tradicionales modelos de regulación, serian además de poco eficaces, agotadores, pues estos actuarían en contra de la composición de cada sistema.

Bajo estas circunstancias, dependiendo de la demanda regulatoria, la posición lógica que debe adoptar un ente regulador es procurar por la autocomposición de sus regulados o, en palabras más precisas, el ente regulador debe propender por la autorregulación de los regulados y esta condición se lograría en gran medida, por medio del encausamiento de los fines de los regulados con los del regulador.

Lo anterior indica, que en la regulación responsiva es fundamental el trabajo colaborativo entre el regulador y el regulado, puesto que los actores regulados son también partícipes en la dinámica regulatoria, pues actúan como si se trataran de elementos asociados. Esto produciría la distribución de la carga regulatoria, así, como la representación eficiente de los intereses (AYRES; BRAITHWAITE, 1992).

Asimismo, la teoría responsiva está vinculada con otro presupuesto y este es el de la biopolítica de Michel Foucault 2007, en donde el filósofo propone que el regulado sea vinculado como un instrumento del poder. En la biopolítica los entes reguladores y los regulados pasan a ser uno solo, pues lo que procura el autor con la biopolítica es descubrir el vínculo del poder sobre la vida y de la vida sobre el poder (FOUCAULT, 2007a).

Empero, es importante destacar que la ligación del poder con la vida no conlleva una connotación negativa, por el contrario, se basa en una relación virtuosa que busca la maximización de los beneficios del regulado (FOUCAULT, 2007a). Esta es una nueva concepción que propone Foucault radicalmente distinta a sus anteriores trabajos basados en su pensamiento del poder disciplinario, en donde concebía al poder como algo negativo, que avasallaba, manipulaba y dominaba (FOUCAULT, 2003, 2007b).

La biopolítica tal vez se trate de una evolución en el pensamiento de Foucault, que buscaba exaltar las bondades que hay en el poder, a través de figuras como el Estado regulador, en donde la ley pasa de ser un artilugio de dominación 
y de muerte, a un elemento fundamental para la vida y para la realización del individuo (ALVES, 2002).

Otro elemento esencial por resaltar en la teoría responsiva, compartido con la biopolítica de Foucault son sus presupuestos económicos. La teoría responsiva al igual que la teoría de la biopolítica poseen fuertes cimientos económicos comunes, este elemento les brinda mayor rigor científico debido a la fortaleza de la evidencia empírica que puede respaldar los estudios de carácter económico.

En cuanto a los aspectos ideológicos relacionados con la economía, la postura que es acogida por las posiciones regulatorias responsivas y por la biopolítica discuerdan con gran parte de los preceptos fundamentales del socialismo, principalmente con aquellos encaminados al debilitamiento del mercado, los cuales se justifican en que el mercado es utilizado como un instrumento de explotación de las masas, en lugar de eso, la postura regulatoria responsiva exalta las bondades que se pueden derivar de la libertad de mercado, enmarcándolo en el contexto del neocorporativismo visto tanto como un eficiente sistema de representación de intereses como un programa de formación, decisión y ejecución de operaciones regulatorias (AYRES; BRAITHWAITE, 1992).

Según lo señalan Braihtwaite y Ayres (1992) algunos de los principales exponentes de la teoría responsiva, ésta parte del ideal de que los regulados son actores virtuosos, no obstante, no se observa en aquellos una masa abstracta y homogénea, en lugar de ello, la teoría responsiva ve en los regulados un conjunto diferenciado, en esa medida los entes reguladores deben proponer una oferta regulatoria que sea eficaz, económicamente lógica y que se ajuste a los requerimientos de reglamentación demandados.

Para conseguir el cometido de ofrecer una variopinta gama de respuestas regulatorias Braihtwaite y Ayres (1992) proponen ordenar la actuación del ente regulador en dos estructuras piramidales, una de sanciones y otra de estímulos, las cuales, funcionan en orden escalonado y se complementan de tal manera que una vez agotado un estímulo, siga otro de mayor atractivo, o en su defecto, agotados los estímulos se impongan sanciones cada vez de mayor gravedad, de no ser que la conducta amerite una consecuencia más gravosa de forma directa.

De este modo, la teoría responsiva propone que existen diferentes ofertas regulatorias para diferentes actores en determinada agrupación social, considerando de esta forma, tanto aquellos que son actores virtuosos y se guían por decisiones lógicas como aquellos que no se guíen por este tipo de decisiones.

Es así como inicialmente en el modelo de regulación responsiva se apela en principio por la autocomposición de los regulados, si esto no es suficiente, se procurará encausar el comportamiento del actor por medio de un atractivo motivacional que estimule su interés racional, en caso de fallar, se procederá con amenazas disuasivas, posteriormente con sanciones y, finalmente, si nada de esto 
produce el efecto regulatorio deseado, se procede con algún tipo de incapacitación como el retiro de la licencia o de los estatutos en caso de ser una compañía privada (AYRES; BRAITHWAITE, 1992). Lo que quiere decir, que la teoría responsiva de ninguna manera propone un ente regulador débil, por el contrario, lo que propone que un ente regulador debe estar fortalecido y especializado.

Luego de realizar diferentes análisis en torno al caso Sepúlveda, de examinar algunas de las falencias que presenta el sistema democrático en Colombia y estudiar parte de los principales fundamentos de la teoría responsiva, se presentan los siguientes aportes, que según el modelo regulatorio responsivo podrían contribuir al fortalecimiento de la democracia colombiana.

\section{Aportes de la teoría responsiva para el fortalecimiento de la democracia colombiana}

Con base en los fundamentos de la teoría responsiva se presentan los siguientes aportes que pueden contribuir al fortalecimiento de la democracia en Colombia:

- En la teoría responsiva se postula una forma de regulación dinámica y flexible, que se ajuste a las cambiantes condiciones de regulación, por ello, con esta teoría se busca que los esfuerzos regulatorios sean invertidos acorde con cada circunstancia específica, de este modo, para el caso en estudio, con el fin de controlar la influencia que es ejercida de forma irregular sobre la decisión de voto de los ciudadanos, se debe procurar, en primera medida, que los programas de trabajo de las campañas políticas se autorregulen, a través de mecanismos como la responsabilidad social e incentivos que los lleven a encauzar su comportamiento de forma autónoma, de no funcionar, los órganos reguladores deben apelar a mecanismos coercitivos cada vez más fuertes y, de no conseguir la conducta esperada por medio de estos métodos, se deben aplicar mecanismos de incapacitación del grupo de trabajo de la campaña política, para garantizar efectivamente la protección de los derechos de los ciudadanos en espacios electorales.

- Es fundamental que sea evaluado con mayor grado de profundidad el nivel de eficacia de la normativa y de las instituciones electorales en Colombia $\mathrm{y}$, de ser necesario, que se reevalúe el sistema normativo electoral, de tal modo que se incluyan en el ordenamiento medidas directas e indirectas que protejan con mayor eficacia el orden electoral y potencialicen los instrumentos que tienen los organismos reguladores para actuar $\mathrm{y}$, de esta 
forma, se fortalezca el poder coercitivo con que cuentan estos organismos, puesto que la teoría responsiva defiende el las entidades reguladoras fortalecidas.

- Frente a la participación política en redes sociales, es indispensable que las entidades reguladoras apoyen sus acciones en los intermediarios de Internet que cumplen entre otros roles el de dispensar las plataformas de redes sociales para el intercambio de la información como lo son Facebook, Twitter o What's app, pues estos actores son fundamentales en los procesos regulatorios en la Internet, en razón a ello, deben ser direccionados a coadyuvar a las entidades reguladoras a integrar estrategias de incentivos y castigos, siempre y cuando se respeten con los derechos y garantías de los ciudadanos en los espacios digitales (las redes sociales también serían fundamentales en los procesos de educación y formación en espacios digitales).

\section{Conclusiones}

Como resultado del análisis de los actos de hacking que fueron objeto de estudio en esta investigación, se puede concluir que a pesar de la amplia estructura del aparato burocrático colombiano en aspectos electorales, no se produjo una eficaz acción del Estado para contrarrestar la influencia de los hackers, por el contrario, lo que se logró establecer fue que estos actores operaron en contra del orden electoral con gran libertad, de igual modo, se evidenció que han sido pocos los eventos en donde se ha impuesto responsabilidad por la comisión de delitos que atenten contra los mecanismos de participación democrática, ni se ha resarcido de alguna manera el daño ocasionado por dichos hechos.

La ausencia de respuesta efectiva del Estado frente a la protección del orden democrático refleja tres estadios posibles que deben ser atendidos y estudiados con mayor profundidad para que sea fortalecida en algunas de sus principales falencias la democracia en Colombia, estos son, en primer lugar, que las instituciones que garantizan los derechos electorales no están cumpliendo con su función satisfactoriamente, en segundo lugar, estos organismos reguladores no cuentan con los instrumentos suficientes para garantizar la conservación del sistema electoral o los mismos no son eficientes, en un último estadio, se puede inferir que la normativa electoral que actualmente rige en materia electoral (por lo menos para el ámbito que fue objeto de estudio en esta investigación), no está cumpliendo cabalmente con los fines constitucionales para los cuales fue instituida, pues de serlo, hubiese sido aplicada con mayor efectividad para reivindicar de este tipo de gravosas transgresiones. 
Esta inacción por parte de las autoridades públicas podría ser traducida como una omisión con relación a la obligación que tiene Estado Colombiano de impartir justicia y de proteger los derechos humanos de los ciudadanos, más aún, cuando se trata de un asunto tan esencial para una democracia como lo es el derecho de sufragio, pues es de destacar, que al finalizar el año 2017 (2 años después de la condena de del hacker), no ha sido socializado por el Estado de Colombia ningún programa, plan, estrategias o política pública que tenga como objetivo garantizar que casos como el de Sepúlveda se repitan en el futuro.

Puede que las nuevas tecnologías de la información y de la comunicación constituyan diferentes oportunidades tanto de fortalecimiento como de riesgo para la democracia, no obstante, lo que se refleja en este estudio tras el silencio que se produjo posterior a la condena de Sepúlveda por delitos contra la seguridad de la información y contra la seguridad del Estado, es que existe un alto nivel de desconocimiento en la sociedad civil con relación a sus derechos políticos y su ejercicio a través de los medios de comunicación digital, como lo son las redes sociales. Es indispensable que se adelanten programas de formación y educación en cultura digital para evitar abusos.

Estas diferentes falencias, como se analizó en la investigación, pueden ser en cierto grado fortalecidas a través de la implementación de postulados de la teoría responsiva en el tradicional modelo regulatorio colombiano, pues entre otros aspectos, la teoría responsiva propone el fortalecimiento de las entidades regulatorias, la producción de una oferta regulatoria acorde con las circunstancias específicas demandadas, la integración de los regulados en el proceso regulatorio $\mathrm{y}$, en general, un modelo de regulación con carácter dinámico, asociativo y flexible, elementos que podrían contribuir notablemente a la protección de los derechos humanos de los ciudadanos y, en consecuencia, al fortalecimiento de la democracia.

\section{Referencias}

ALVES, M. Michel foucault e o direito. São Paulo: Max Limonad, 2002.

ANTUNES, J. O direito como sistema autopoiético. [s.1.]: Fundação Caoluste Gulbenkian, 1989.

AYRES, I.; BRAITHWAITE, J. Responsive Regulation. Oxford: Oxford sociallegal studies, 1992.

BONAÑO, L. Las redes sociales como fuentes de informacion. Sevilla: 
Universidad de Sevilla, 2015.

BOTERO, C. et al. Libertad de expresión: debates, alcances y nueva agenda.

Quito: Organizacion de las Naciones Unidas para la educacion, 2011.

BUSINESSWEEK, B. Cómo Hackear una Elección. 4 abr. 2016.

CARBONELL, M. La libertad de expresión en materia electoral. Mexico: Tribunal Electoral del Poder Judicial de la Federacion, 2008.

CASTELLS, M. Comunicación y poder. Barcelona: Alianza Editores, 2009.

CENTRO NACIONAL DE MEMORIA HISTÓRICA. Colombia: Memorias de guerra $\quad \mathbf{y}$ dignidad. Disponible en: 〈http://www.centrodememoriahistorica.gov.co/micrositios/informeGeneral/estadisticas.html〉. Acesso em: 14 jan. 2018.

COMANDUCCI, P. Formas de (neo) constitucionalismo: un análisis metateórico. Isonomía, n. 16, p. 89-112, 2002.

COLOMBIA. Ley 599 del año 2000. Por la cual se expide el Código Penal. Diario Oficial. Diario Oficial No. 44.097 del 24 de julio del 2000. Bogotá, D.C. Disponible en: 〈http://www.secretariasenado.gov.co/senado/basedoc/ley_0599_2000.html〉 Acceso en: 08 de enero de 2018.

COLOMBIA. Constitución Política de Colombia del año 1991. Gaceta Constitucional No. 116 de 20 de julio de 1991. Bogotá, D.C. Disponible en: 〈http://www.secretariasenado.gov.co/senado/basedoc/constitucion_politica_1991.html〉 Acceso en: 12 de enero de 2018.

DUTTON, W. H. et al. Freedom of expression: The changing legal and regulatory ecology shaping the internet. [s.1.] UNESCO, 2010.

EL PAÍS. En 54 años, 220 mil personas han muerto por el conflicto armado en Colombia. 24 jul. 2013.

EL TIEMPO. FINANZAS DE LAS FARC: UNA GUERRA EN PAÑALS Archivo Digital de Noticias de Colombia y el Mundo desde 1.990 eltiempo.com. Disponible en: 〈http://www.eltiempo.com/archivo/documento/MAM1308383>. Acesso em: 9 dez. 2017.

EL TIEMPO. Rumores del plebiscito por la paz. 2016.

FGV DAPP. Robôs, redes sociais e política no Brasil. 2017.

FISCALIA GENERAL DE LA NACIÓN. Condenado hacker Andrés Sepúlveda por interceptar a negociadores de paz en La Habana. 
Disponible en: <http://www.fiscalia.gov.co/colombia/noticias/condenado-hacker-andressepulveda-por-interceptar-a-negociadores-de-paz-en-la-habana/>. Acesso em: 4 ago. 2017.

FOUCAULT, M. Vigilar y Castigar. Argentina: Siglo veintiuno editores Argentina, 2003. v. 53

FOUCAULT, M. Nacimiento de la biopolítica curso: Collège de France. In: SENELLART, M.; FONTANA, A.; FRANÇOIS, E. (Eds.). . Mexico: Fondo de Cultura Económica, 2007a.

FOUCAULT, M. Historia de la sexualidad. Vol 1. Voluntad de saber. [s.1.] Siglo veintiuno editores Argentina, 2007b.

FRAZÃO, A. Premissas para a reflexão sobre a regulação da tecnologia | JOTA. JOTA, 2017.

GANDÁSEGUI, M. A. et al. Estados Unidos y la nueva correlacion de fuerzas internacional. Buenos Aires: CLACSO, 2016.

INSTITUTO NACIONAL DE TECNOLOGIAS DE LA COMUNICACIÓN. ¿Qué es (y qué no) un hacker? [s.l: s.n.].

LA REPÚBLICA. El No ha sido la campaña más barata y más efectiva de la historia. 4 out. 2016.

LEGISLATIVO, O. Normas electorales. Boletín del Instituto de Ciencia Politica Hernan Echavarria Olozaga, n. 146, p. 1-4, 2009.

LUHMANN, Niklas. El derecho de la sociedad. [s.1.] 2003.

MITNICK, K. D; SIMON, W. L. El arte de la Intrusión. La Verdadera Historia de las Hazañas de Hackers, Intrusos e Impostores. México D.F.: Alfaomega Grupo Editor, 2007.

NIETO, J. La Libertad y la democracia como instrumentos de dominación. p. 239-260, 2010.

PANTOJA, A. Los nuevos medios de comunicación social. Las redes sociales, v. 12, n. 1988-8430, p. 218-226, 2011.

RAZÓN PÚBLICA. Las redes sociales y el plebiscito. 21 ago. 2016.

ROBINSON, J. P. Public opinion during the watergate crisis. Comunication Research, v. 1, p. 1-15, 1974.

SEMANA. Las cinco mentiras del plebiscito que circularon por WhatsApp. 29 
set. 2016.

CIFUENTES, J. E. S. La autorregulación comunicacional en los procesos de votación: una respuesta jurídico-politica al problema del "backing electoral" practicado a través de las redes sociales. Un análisis del caso colombiano y de su repercusión en América Latina. The Law, State and Telecommunications Review, Brasilia, v. 10, n. 2, p. 15-36, October 2018. [DOI: https://doi.org/10.26512/1str.v10i2.21491] 